\title{
Wenn Lernsituationen zu \\ Leistungssituationen werden: \\ Untersuchung zur Fehler- \\ kultur in einer Videostudie
}

\section{Lena Meyer, Tina Seidel und Manfred Prenzel}

Der vorliegende Beitrag zeigt, wie Aspekte einer Fehlerkultur systematisch mittels $V i$ deoanalysen erfasst und Unterschiede zwischen Schulklassen abgebildet werden können. Im Zentrum steht dabei die in Deutschland häufig problematisierte Vermischung von Lern- und Leistungssituationen. Die Ergebnisse aus den durchgeführten Videoanalysen verdeutlichen, dass internationale Kriterien für die Zuverlässigkeit der Verfahren in allen Bereichen erfüllt und darüber hinaus durch die Sicht der Schülerinnen und Schüler gestützt werden. Damit liegt ein Instrumentarium vor, das einen Aspekt des Bedingungsgeflechtes von Fehlerkultur im Unterrichtsvideo bestimmen und konkrete relevante Unterrichtssituationen kennzeichnen kann. Es eröfnen sich reichhaltige Möglichkeiten für vertiefende Analysen. Anhand zweier Fallanalysen wird das Potenzial aufgezeigt.

\section{Einleitung}

Bis vor kurzem wurden Fehler im schulischen Lernen verteufelt, vermieden und oft ignoriert. Seit TIMSS und PISA werden Fehler jedoch häufiger als notwendiger Bestandteil verstehensorientierter Lernprozesse diskutiert. Dass Fehler heute neu gesehen werden und dass über eine "Fehlerkultur» gesprochen wird, ist ein Verdienst von Fritz Oser und seiner Arbeitsgruppe. Sie hat zuerst Untersuchungen über Fehler aus anderen Forschungsfeldern aufgearbeitet (Oser \& Hascher, 1997) und danach in ausgedehnten Forschungsarbeiten gezeigt, dass Fehler produktiv genutzt werden können, wenn sie innerhalb formativer Lernprozesse auftreten und gezielt behandelt werden (Oser \& Spychiger, 2005). Das setzt zum Beispiel voraus, Übungs- und Kontrollphasen im Unterricht für die Schülerinnen und Schüler nachvollziehbar und klar voneinander zu trennen (Spychiger, Oser, Hascher, \& Mahler, 1999). Wie man mit einem Beobachtungsverfahren die Vermischung von Lern- und Leistungssituationen erfassen kann, stellt dieser Beitrag vor. Er berichtet über ein Schätzverfahren, das im Rahmen von Videoanalysen eingesetzt wurde. Mit Hilfe des Verfahrens sollen Infor- 
mationen über das Lernklima einer Klasse sowie über die Fehlerkultur im Unterricht gewonnen werden.

\section{Theoretischer Hintergrund}

\section{Lernen aus Fehlern}

Untersuchungen zu Fehlern finden derzeit in verschiedenen Kontexten statt. Je nach Kontext und Fragestellung variiert das Verständnis des Begriffs «Fehler» und rücken unterschiedliche Funktionen von Fehlern in den Blickpunkt. Im Kontext der Lehrerausbildung nutzt zum Beispiel Spychiger (2003) Fehler von Schülerinnen und Schülern, um deren Lernprozesse zu diagnostizieren und um gemeinsam Anknüpfungspunkte für Lernprozesse zu erarbeiten. Aus einer ähnlichen Perspektive unterscheiden Zapf, Frese und Brodbeck (1999) in Arbeitszusammenhängen zwei Bedeutungen von Fehlern: Sie zeigen die Grenzen kognitiver Verarbeitungsprozesse an und gestatten es, auf kognitive Prozesse zurück zu schliessen. Für das schulische Lernen betonen Oser und seine Arbeitsgruppe die Funktion des Fehlers, Anlass zu geben, «Schutzwissen» und "Abgrenzungswissen» aufzubauen (Oser \& Spychiger, 2005; Oser \& Hascher, 1997). Ein Lernen aus Fehlern kann so zum Aufbau «negativen Wissens» führen. Fehler helfen dabei zu klären, wie ein Sachverhalt nicht ist oder wie etwas nicht getan wird.

In all diesen Kontexten bleibt jeweils zu klären, was einen Fehler ausmacht und worin er besteht. Damit verbunden sind immer Kriterien oder Normen, die festgelegt werden müssen, um Abweichungen zu erfassen und um Fehler zu identifizieren (Kobi, 1994; Oser \& Hascher, 1997). Spychiger (2003) nutzt in der bereits angesprochenen Studie über den Umgang von Lehramtsstudierenden mit Schülerfehlern Expertenurteile als «Kriterium», anhand derer das Verhalten der Studierenden als fehlerhaft oder nicht fehlerhaft bewertet wird. Zapf et al. (1999) dagegen verwenden Definitionen von Zielen und Teilzielen, um daran abweichende Ergebnisse als Fehler zu klassifizieren und um Reaktionen auf Fehler im Sinne eines Fehlermanagements zu bewerten.

Auch im schulischen Lernen spielen Normen eine entscheidende Rolle, um Fehler zu identifizieren. Doch diese Normen unterscheiden sich je nachdem, ob es sich um Lern- oder Leistungssituationen handelt. In Leistungssituationen ist das richtige Ergebnis meist klar definiert und wird für die Schülerinnen und Schüler spätestens bei der Beurteilung sichtbar und nachvollziehbar. In Lernsituationen sind entsprechende Normen jedoch sehr viel weniger deutlich und greifbar. Neben das Kriterium der Richtigkeit treten andere Aspekte, die mit dem Vorwissen der Lernenden zu tun haben, aber auch mit bestimmten Unterrichtsformen, die individuelle, zum Teil explorierende Zugänge vorsehen und die nicht immer unter Aufsicht erfolgen. In Hinblick auf den Lernprozess können sich manche Fehler als mehr oder weniger produktiv erweisen (Oser \& Spychiger, 2005; Seidel \& Prenzel, 2003). 
In Leistungssituationen gilt es Fehler zu vermeiden beziehungsweise diese rechtzeitig zu entdecken und zu korrigieren. Die Rückmeldung von Fehlern nach Prüfungs- oder Leistungssituationen bedeutet für Schülerinnen und Schüler, auf Misserfolge aufmerksam gemacht zu werden (Weinert, 1999). In der Schule ergibt sich jedoch immer wieder eine besondere Konstellation, in der Lern- und Leistungssituationen vermischt werden, nämlich dann, wenn die Schülerinnen und Schüler in Lernsituationen damit rechnen müssen, dass ihre Beiträge oder ihre Mitarbeit in die Leistungsbewertung eingehen (z.B. als mündliche Note). Zur Leistungssituation werden Lernsituationen auch dann, wenn Schülerinnen und Schüler sich nach einem Beitrag ignoriert, bloss gestellt oder beschämt fühlen. Eine Konsequenz im Verhalten der Schülerinnen und Schüler liegt darin, dass sie Fehler vermeiden bzw. sich nur aktiv beteiligen, wenn sie sicher sind, dass ihr Beitrag «richtig» ist. Das Lernen der Schülerinnen und Schüler findet damit nur eingeschränkt statt. Fehler und Fehlkonzepte werden nicht genutzt, um positives wie negatives Wissen aufzubauen. Wenn die Schülerinnen und Schüler darüber hinaus implizite oder explizite Bewertungen oder gar Beschämungen befürchten müssen, wird die Fehlerkultur durch ungünstige affektiv-motivationale Bedingungen belastet. Während das Lösen von Fehlern mit Kompetenzerleben und Formen selbstbestimmter Lernmotivation einher geht (Hascher \& Oser, 1996), sind Fehler in Leistungssituationen mit negativen Attributionen verbunden (Weinert, 1999). Wie die Vermischung von Lern- und Leistungssituationen sich auf affektiv-motivationale Faktoren auswirkt und wie entsprechende Bedingungen von vermischten Lern-Leistungssituationen im Unterrichtsvideo analysiert werden können, stellen wir in diesem Artikel vor.

\section{Beobachten von Fehlerkultur im Unterrichtsvideo}

Das Unterrichtsvideo eignet sich sehr gut zur Beobachtung von Fehlerkultur. Es bietet die Möglichkeit, systematische Analysen wiederholt durchzuführen, um so Perspektiven miteinander zu kombinieren und Kodierungen von mehreren Personen durchführen zu lassen.

Die Untersuchung der Fehlerkultur durch einen Situationsansatz (Spychiger, Oser, Hascher \& Mahler, 1997; Oser \& Spychiger, 2005), der anhand eines Vierfelder-Schemas vier Situationstypen durch die Unterscheidung von Lernklima und Lernorientierung beschreiben lässt, ist von Videoanalysen ausgegangen und hat seinerseits viele Impulse für weitere und ausgefeiltere Videoanalysen gegeben. So konnte zum Beispiel Büeler (2000) durch Fehlerflussanalysen die nonverbalen Reaktionen von Lehrkräften auf Fehler in förderliche und hinderliche Verhaltensmuster differenzieren. Schoy-Lutz (2005) erfasste durch ihre Fehlersituationsanalyse kognitive und emotionale Bedingungen von Fehlerkultur im Mathematikunterricht. Heinze (2004) untersuchte im Mathematikunterricht in Deutschland Beziehungen zwischen dem Fehlertyp, der reagierenden Person in der Fehlersituation und der Art der Reaktion. Ein Hauptergebnis seiner Untersuchung aber war, dass in den von ihm untersuchten Klassen insgesamt 
nur sehr wenige Fehler - im Mittel weniger als fünf pro Stunde - auftraten und damit eine systematische Analyse der Reaktionen auf Fehlersituationen nur eingeschränkt stattfinden konnte.

Aus methodischer Sicht wirft die geringe Anzahl von Fehlern, die in deutschen Unterrichtsvideos identifiziert werden können, etliche Probleme auf. Ein Kennzeichen des deutschen Unterrichts ist es gerade, dass fast ausschliesslich positive Beispiele oder Elemente des Lernstoffs behandelt werden. Fehler werden somit selten sichtbar. Ein anderes Hemmnis für die videogestützte Beobachtung ist das sogenannte «Bermuda-Dreieck»: Falsche oder unerwünschte Beiträge der Lernenden werden von der Lehrperson übergangen. Bei der Umsetzung dieses Unterrichtsgeschehens in Beobachtungsverfahren wirft diese Situation wiederum zahlreiche Probleme auf, die vor allem mit der Normierung von Fehlern zu tun haben. Bei der Kodierung ist häufig kaum zu entscheiden, wann es sich bei einem Schülerbeitrag, auf den nicht reagiert wird, um einen Fehler handelt. Darüber hinaus ist die qualitative Einschätzung der Wirkung solcher «BermudaSituationen» kritisch, weil der Effekt eng mit der Qualität der Fehlerkultur verknüpft ist (Oser \& Hascher, 1997).

Probleme für die Videobeobachtung ergeben sich zusammenfassend vor allem dann, wenn das Nicht-Auftreten eines Verhaltens (z.B. einer Fehlerermunterungsdidaktik im Sinne von Oser \& Spychiger, 2005) zwar theoretisch relevant ist, aber im Video nicht oder nur sehr pauschal abgebildet werden kann. Beobachtbar sind jedoch solche unterrichtlichen Muster, Aktivitäten und Verhaltensweisen, die problematische Ausgangsbedingungen für einen produktiven Umgang mit Fehlern darstellen. Das Vermeiden von Fehlern als Ausdruck einer wenig lernförderlichen Fehlerkultur muss dann über das Verhalten der Lehrpersonen sowie der Schülerinnen und Schüler beschrieben werden. Ein Ansatz für die Erfassung problematischer Ausgangsbedingungen mittels Videoanalysen kommt weiter unten zur Darstellung.

\section{Routinierte Handlungsmuster: Unterrichtsskripts}

Die Analyse von Unterrichtsskripts im Video stellt Informationen über Handlungsmuster bereit, die zur Routine geworden sind (Seidel, 2003). Sie sind Bestandteil der Unterrichtskultur einer Klasse und bieten unterschiedliche Zeitfenster für Aktivitäten an, die das Lernen aus Fehlern mehr oder weniger wahrscheinlich machen. Folgende Ergebnisse von Videoanalysen liefern aussagekräftige Kriterien für das Ermöglichen eines produktiven Umgangs mit Fehlern.

Kuntze und Reiss (2004) stellten für den Mathematikunterricht fest, dass die Lehrerfragen auf einem wenig anspruchsvollen kognitiven Niveau ansetzen. Die Antworten der Schülerinnen und Schüler dienen vorwiegend dazu, die Aussagen der Lehrkraft zu ergänzen. Auch im deutschen Physikunterricht können Muster von Unterrichtsaktivitäten identifiziert werden, die als stärker lehrerzentriert oder als stärker schülerzentriert bezeichnet werden können. Im Physikunterricht der 9. Jahrgangsstufe wurden hohe Anteile lehrerzentrierter Aktivitäten identifi- 
ziert (Seidel \& Prenzel, 2004). Zu diesen Aktivitäten zählen Lehrervortag, Diktat / Hefteintrag und das Klassengespräch. In nur wenigen Klassen liegt der Anteil lehrerzentrierter Aktivitäten unter 50 Prozent der Unterrichtszeit (kumulierte Prozentwerte). Hierbei ist die Begleitung des Lernens eng geführt (Seidel, 2003). Die Schülerinnen und Schüler nehmen eine passive Rolle ein und die Lehrpersonen stellen zum grössten Teil geschlossene Fragen, die meistens genau auf eine gewünschte Antwort abzielen. Eine ähnliche Qualität weist die Lernbegleitung in Schülerarbeitsphasen auf. Obwohl in diesen Phasen eine stärkere Schülerorientierung zu vermuten wäre, geben auch hier die Lehrpersonen die meisten Schritte rezeptartig vor, nehmen sich selbst wenig zurück und geben selten individuelle Rückmeldungen (Kobarg, 2004). In einem Unterricht, in dem die Lehrpersonen im Mittelpunkt aller Aktivitäten stehen und der Lernstoff kleinschrittig behandelt wird, ist der konstruktive Umgang mit Fehlern zwar nicht unmöglich, jedoch sind die Bedingungen für formative Lernaktivitäten und angstfreien probierenden Aktivitäten ungünstig.

\section{Vermischung von Lern- und Leistungssituationen}

$\mathrm{Ob}$ es im Unterricht zur probierenden Auseinandersetzung mit dem Lerngegenstand kommt, ist neben den Interaktionen zwischen Lehrenden und Lernenden eng mit dem Lernklima in der Klasse verbunden (Hascher, 2004). Ein negatives Lernklima kann dabei Ausdruck bzw. Resultat einer systematischen Vermischung von Lern- und Leistungssituationen sein (Götz, Zirngibl \& Pekrun, 2004). Entscheidend für die Einschätzung der Lernklimas ist die individuelle Wahrnehmung der Schülerinnen und Schüler. Erleben diese einen Unterricht als Bewertungssituation, kommen individuelle Lern- und Leistungsemotionen zum Tragen (Pekrun, 2000).

Das Lernklima in der Klasse stellt damit ein Resultat einer systematischen Vermischung von Lern- und Leistungssituationen dar. Günstigere bzw. ungünstigere unterrichtliche Ausgangsbedingung für ein Lernen an Fehlern sind mittels Videoanalysen erfassbar. Gleichzeitig sind die Analysen auf die Erfassung vorherrschender Unterrichtsbedingungen in einer Klasse beschränkt. Ursache-Wirkungsanalysen sind darüber allerdings schwerer fassbar. Dies trifft vor allem dann zu, wenn einzelne Unterrichtsstunden analysiert werden. Trotzdem gibt es Belege dafür, dass auch über kurze Zeitfrequenzen Unterrichtsroutinen zuverlässig erfasst werden und vorherrschende Unterrichtsbedingungen in einzelnen Stunden Ausdruck einer stabilen Unterrichtskultur sein können (Seidel \& Prenzel, 2006).

Videoanalysen zur Vermischung von Lern- und Leistungssituationen stellen grundlegende Informationen über das Lernklima und über Ausgangsbedingungen für einen Umgang mit Fehlern bereit. Zentral ist dabei nicht das Auftreten eines Fehlers und die darauf folgenden Reaktionen (anders als bei Oser \& Hascher, 1997; Schoy-Lutz, 2005), sondern das Lernklima im Gesamtverlauf einer Unterrichtsstunde. Zwei Komponenten können hierbei unterschieden werden (Schulmeiss, 2004): 
(a) Ängstlichkeit und Beschämung umfassen negative Bedingungen des Lernklimas, einerseits zwischen der Lehrperson und den Schülerinnen bzw. Schülern, andererseits zwischen den Mitschülerinnen und Mitschülern. Kennzeichnend ist eine angespannte Atmosphäre in der Klasse. Schülerinnen und Schüler, die von der Lehrperson zur Äusserung aufgefordert werden, verhalten sich eingeschüchtert, äussern sich beispielsweise zaghaft (im Frageton) oder verweigern ihre Beteiligung.

(b) Hinweise auf Fehlervermeidung und negative Fehlerreaktion geben Schülerinnen und Schüler, die häufiger Bewertung durch die Lehrperson ausgesetzt sind und das Auftreten von fehlerhaften Äusserungen verhindern oder die Antworten, bei denen sie sich besonders sicher fühlen, deutlich betonen.

\section{Effekte auf das Lernen der Schülerinnen und Schüler}

Aus lern- und motivationstheoretischer Sicht ist zu erwarten, dass sich eine Vermischung von Lern- und Leistungssituationen negativ auf motivationale Prozesse und auf die Entwicklung eines überdauernden Interesses am Lerngegenstand auswirkt (Krapp, 2002). Bisherige Forschungsarbeiten stützen sich allerdings ausschliesslich auf Schülerselbsteinschätzungen und damit auf die individuelle Wahrnehmung durch die Schülerinnen und Schüler. Für die Entwikklung eines Videoanalyseverfahrens wird von Interesse sein, inwieweit die über eine «Aussensicht» ermittelten Indikatoren für die Vermischung von Lern- und Leistungssituationen über die individuellen Einschätzungen der Schülerinnen und Schüler («Innensicht») getragen werden (Seidel, 2003).

\section{Fragestel lungen}

Vor diesem Hintergrund verfolgt der vorliegende Beitrag vier Fragen:

1. Ist das Ausmass von Vermischung von Lern- und Leistungssituationen mittels Videoanalysen beobachtbar?

2. Wie unterscheiden sich zufällig ausgewählte Schulklassen in der Vermischung von Lern- und Leistungssituationen?

3. Gibt es systematische Zusammenhänge zwischen dem Ausmass einer Vermischung von Lern- und Leistungssituationen und der individuellen Wahrnehmung durch die Schülerinnen und Schüler?

4. Wie sieht beispielhaft ein Unterricht aus, der Lern- und Leistungssituationen mehr oder aber weniger vermischt?

Diese Fragestellungen werden mit Hilfe von Beobachtungsverfahren und Daten aus der Videostudie, die wir am Leipniz-Institut für die Pädagogik der Naturwissenschaften («IPN-Videostudie») durchgeführt haben, beantwortet. Als Grundlagen dienen Aufzeichnungen von Unterrichtsstunden aus der Physik. 


\section{Methode}

Die methodischen Verfahren werden im Folgenden kurz dargestellt. Eine detaillierte Beschreibung aller Erhebungs- und Analyseverfahren findet sich im technischen Bericht der IPN Videostudie (Seidel, Prenzel, Duit, \& Lehrke, 2003).

\section{Stichprobe und Design}

An der Studie nahmen 50 deutsche Gymnasial- und Realschulklassen (1249 Schülerinnen und Schüler der 9. Jahrgangsstufe) teil. Die Videoaufzeichnungen $(\mathrm{N}=100)$ fanden in zwei aufeinander folgenden Physikunterrichtsstunden statt. Die beiden einführenden Stunden behandelten die Themen «Linsengesetze» oder «Kraftbegriff».

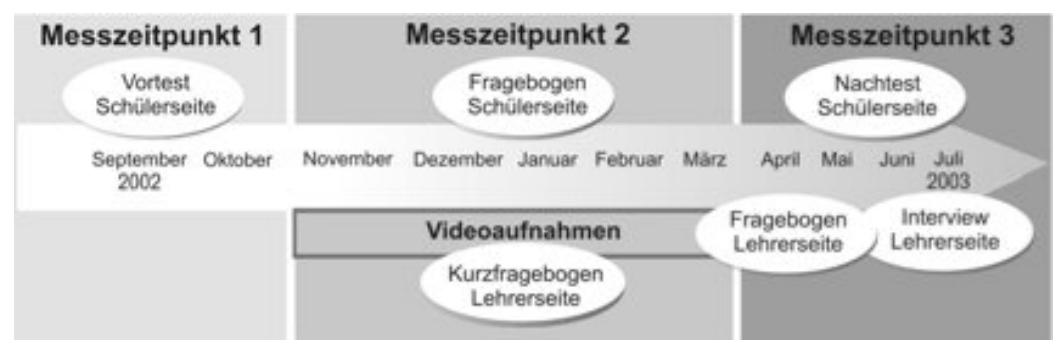

Abbildung 1: Design der IPN Videostudie.

Die Erhebungen wurden im Schuljahr 2002/2003 in vier Bundesländern (Baden-Württemberg, Bayern, Brandenburg und Schleswig-Holstein) durchgeführt. Die Ziehung der Stichprobe erfolgte nach einem stratifizierten Zufallsverfahren durch das Data Processing Centre (DPC) in Hamburg.

\section{Erhebungsmethoden}

Um die Vermischung von Lern- und Leistungssituationen zu erfassen, werden die Videoanalysen (1) durch Daten aus dem Schülerfragenbogen (2) ergänzt. Die Fragebögen erheben direkt nach den aufgezeichneten Unterrichtsstunden die Wahrnehmung unterstützender Lehr-Lern-Bedingungen und die Qualität der Lernmotivation.

(1) Erfassung der Vermischung von Lern- und Leistungssituationen über Videoanalysen: Die Vermischung von Lern- und Leistungssituationen in den Schulklassen wird mittels eines Schätzverfahrens erfasst. Dazu beobachten Kodiererinnen den gesamten Unterrichtsverlauf am Video und schätzen fünf Aspekte ein, die in Figur 2 im Überblick dargestellt sind. Jeder Aspekt greift eine unterrichtliche Bedingung auf, die am Verhalten der Klasse zu beurteilen ist. Eine detaillierte Beschreibung der fünf Aspekte sowie der Kodieranleitungen findet sich bei 
Schulmeiss, Seidel und Meyer (2003). Zur Umsetzung des Verfahrens führen zwei Personen unabhängig voneinander die Einschätzungen durch.

\begin{tabular}{|c|ll|}
\hline 1 & $\begin{array}{l}\text { Angespannte Atmosphäre } \\
\text { Negative Fehlerreaktion }\end{array}$ & $\begin{array}{l}\text { In der Klasse herrscht eine angespannte Atmosphäre } \\
\text { Die Klasse reagiert verächtlich auf fehlerhafte } \\
\text { Äusserungen }\end{array}$ \\
3 & Ängstlichkeit bei Aufforderung & $\begin{array}{l}\text { Schüler und Schülerinnen, die von ihrer Lehrkraft zu } \\
\text { einer Äusserung / Aktivität aufgefordert werden, ohne } \\
\text { sich gemeldet zu haben, sind ängstlich. }\end{array}$ \\
& Fähigkeitsdemonstration & $\begin{array}{l}\text { Die Schülerinnen und Schüler, die sich während des Un- } \\
\text { terrichts äussern, sind bemüht, möglichst fähig zu } \\
\text { wirken. }\end{array}$ \\
& Beschämung vermeiden & $\begin{array}{l}\text { Die Schülerinnen und Schüler, die sich während des } \\
\text { Unterrichts äussern, versuchen falsche Äusserungen zu } \\
\text { vermeiden, um Beschämungen zu entgehen. }\end{array}$ \\
& &
\end{tabular}

Abbildung 2: Aspekte des Schätzverfahrens zur Vermischung von Lern- und Leistungs-situationen durch Videobeobachtung. Antwortformat: $3=$ Trifft $z u, 2=$ Trifft grösstenteils $z u, 1=$ Trifft teilweise $z u, 0=$ Trifft nicht $z u$.

Eine faktorenanalytische Überprüfung der Einschätzungen führte zu folgendem Ergebnis: Die drei Aspekte (1) Angespannte Atmosphäre, (3) Ängstlichkeit bei Aufforderung und (5) Beschämung vermeiden, bilden einen Faktor, der unter «Ängstlichkeit und Beschämung» zusammengefasst wird. «Fehlervermeidung und negative Fehlerreaktion» stellen einen zweiten Faktor dar, der durch die beiden Aspekte (2) Fehlerreaktion und (4) Fähigkeitsdemonstration bestimmt ist.

(2) Erfassung der individuellen Wahrnehmung und der Lernmotivation der Schülerinnen und Schüler: Direkt im Anschluss an die Videoaufzeichnungen wurden die Schülerinnen und Schüler zur individuellen Wahrnehmung des Unterrichts und zur Qualität ihrer Lernmotivation befragt. Eine Darstellung der Skalen und Kennwerte findet sich bei Rimmele et al. (2005). Im Beitrag gehen die Skalen zur Lernmotivation und zur Wahrnehmung unterrichtlicher LehrLern-Bedingungen ein. In der IPN Videostudie werden sechs unterstützende Lehr-Lern-Bedingungen erfasst. Diese umfassen die von den Lernenden wahrgenommene inhaltliche Relevanz, die Instruktionsqualität, das inhaltliche Interesse der Lehrperson, die soziale Einbindung und die Kompetenz- und Autonomieunterstützung. Im Bereich der Lernmotivation werden fünf Varianten differenziert: amotiviert, external, introjiziert, identifiziert und intrinsisch/interessiert motiviert. 


\section{Ergebnisse}

Die Darstellung der Ergebnisse erfolgt entsprechend der vier Fragestellungen. Dazu wird zunächst geklärt, ob aus methodischer Sicht eine zuverlässige Erfassung der Vermischung von Lern- und Leistungssituationen gewährleistet ist. Darauf aufbauend erfolgt eine Darstellung der deskriptiven Ergebnisse in den untersuchten Schulklassen. Die Ergebnisse der Videoanalysen spiegeln wir mit den Einschätzungen der Schülerinnen und Schüler. Abschliessend werden zwei Fallanalysen vorgestellt, um die Art der Vermischung von Lern- und Leistungssituationen zu vertiefen und das Zusammenspiel von Ergebnissen der Videoanalysen und der Schülerselbsteinschätzungen zu illustrieren.

\section{Systematische Analyse von Fehlerkultur im Unterrichtsvideo}

Im ersten Abschnitt wird geklärt, ob eine zuverlässige Erfassung der Vermischung von Lern- und Leistungssituationen mittels Videoeinschätzungen möglich ist. Dazu greifen wir auf die fünf Aspekte des Videoinstruments zurück, die anhand der beiden Skalen «Ängstlichkeit und Beschämung» sowie "Fehlervermeidung und negative Fehlerreaktion" gruppiert werden. In Tabelle 1 sind die deskriptiven Informationen und die Zuverlässigkeitswerte der Kodierung durch zwei unabhängige Kodiererinnen aufgeführt. Die Mittelwerte der Aspekte auf der vierstufigen Antwortskala (von $0=$ Trifft nicht zu bis $3=$ Trifft zu) sind insgesamt gering ausgeprägt (Mittelwerte von 0.18 bis 1.15), verteilen sich jedoch über die gesamte Spannweite möglicher Einschätzungen (Minimum von 0 bis Maximum von 3).

Tabelle1: Deskriptive Kennwerte und Zuverlässigkeit der Einschätzungen zur Vermischung von Lern- und Leistungssituationen ( $N=100$ Unterrichtsstunden).

\begin{tabular}{|lccccccc|}
\hline & M & SD & Min & Max $\begin{array}{c}\text { Über- } \\
\text { einstim- } \\
\text { mung }\end{array}$ & $\begin{array}{c}\text { Kohens } \\
\text { Kappa }\end{array}$ & $\begin{array}{c}\text { G- } \\
\text { Koeffi- } \\
\text { zient }\end{array}$ \\
\hline Ängstlichkeit und Beschämung & & & & & & & \\
Angespannte Atmosphäre (1) & 0.29 & .48 & 1 & 3 & 86.0 & .65 & .85 \\
Ängstlichkeit (3) & 0.51 & .52 & 1 & 3 & 85.7 & .76 & .90 \\
Beschämung vermeiden (5) & 1.15 & .61 & 0 & 3 & 87.7 & .77 & .90 \\
\hline Fehlervermeidung und negative Fehlerreaktion & & & & & & \\
Negative Fehlerreaktion (2) & 0.27 & .43 & 2 & 3 & 94.9 & .87 & .93 \\
Fähigkeitsdemonstration (4) & 0.18 & .39 & 1 & 3 & 93.9 & .79 & .90 \\
\hline
\end{tabular}


Als Kriterien für die Zuverlässigkeit werden drei Masse herangezogen: Die prozentuale direkte Übereinstimmung, Cohens Kappa und der Generalisierbarkeitskoeffizient. Die prozentualen Übereinstimmungen belegen mit Werten von $85 \%$ bis $95 \%$ sehr gute Zuverlässigkeiten. Die weiteren Kennzahlen berücksichtigen darüber hinaus Anteile zufälliger Übereinstimmungen (Cohens Kappa) und die Bestimmung der Anteile an Unterschieden im Ausmass der Vermischung durch Variablen der Beobachterinnen oder der tatsächlichen Varianz des Unterrichts (G-Koeffizient). Für vier der fünf Aspekte liegen die Cohens Kappa Werte über .70 und entsprechen somit internationalen Standards (Bortz \& Döring, 2002). Der Generalisierbarkeitskoeffizient (relativ) zeigt für alle Aspekte ebenfalls sehr gute Kennwerte (>.85), weswegen angenommen werden kann, dass durch die Unterschiede zwischen den Unterrichtsstunden deutlich mehr Anteile in die Variationen der Messung einfliessen als durch Komponenten der beiden Kodiererinnen (Clausen, Reusser \& Klieme, 2003; Kobarg \& Seidel, 2005).

Zusammenfassend verweisen die statistischen Analysen auf eine sehr hohe Zuverlässigkeit des Videoanalyseverfahrens. Die Unterrichtsstunden konnten sehr gut hinsichtlich der Ausprägung von "Angst und Beschämung» und «Fehlervermeidung und negative Fehlerreaktion» kodiert werden. Damit lässt das Verfahren eine Beschreibung und Unterscheidung der Schulklassen bezogen auf das Ausmass der Vermischung an Lern- und Leistungssituationen zu. Im Weiteren wird deshalb der Frage nachgegangen, wie sehr sich die untersuchten Schulklassen in der Vermischung von Lern- und Leistungssituationen unterscheiden.

$\mathrm{Zu}$ den Unterschieden zwischen den Schulklassen in der Vermischung von Lernund Leistungssituationen: Die Darstellung der Unterschiede zwischen den 50 untersuchten Schulklassen erfolgt anhand der beiden Bereiche «Ängstlichkeit und Beschämung» und "Fehlervermeidung und negative Fehlerreaktion». Dazu sind in zwei Abbildungen (Figur 3 und 4) die Ergebnisse der Videoeinschätzungen pro Klasse dargestellt. Die Werte beziehen sich auf den Mittelwert der Einschätzung beider Kodiererinnen. Zudem wurden die Einschätzungen zu den beiden aufgezeichneten Unterrichtsstunden in einem Mittelwert zusammenfasst. Die Einschätzungen beziehen sich auf einen Bereich von $0=$ Trifft nicht zu bis $3=$ Trifft $\mathrm{zu}$.

Die Ergebnisse der Videoanalysen zeigen folgendes Bild: In allen Klassen kamen Situationen vor, in denen sich die Schülerinnen bzw. Schüler ängstlich und/oder beschämt verhalten haben. Nur in sechs der insgesamt 100 Unterrichtsstunden wurde von den Kodiererinnen keine einzige Situation erkannt, in der ein ängstliches Verhalten der Schülerinnen und Schüler vorkam. Darüber hinaus macht Abbildung 3 deutlich, dass die Ausprägungen von Ängstlichkeit und Beschämung insgesamt niedrig eingestuft wurden. Der Mittelwert der Kodierungen über alle Klassen liegt bei 0.65 (bei einem Bereich zwischen 0 und 3). Trotz dieser niedrigen Ausprägungen werden dennoch einige Unterschiede zwischen den 50 Schulklassen deutlich. In 10 Klassen sind die Ausprägungen unter dem Mittelwert ( $M=0.65)$. 


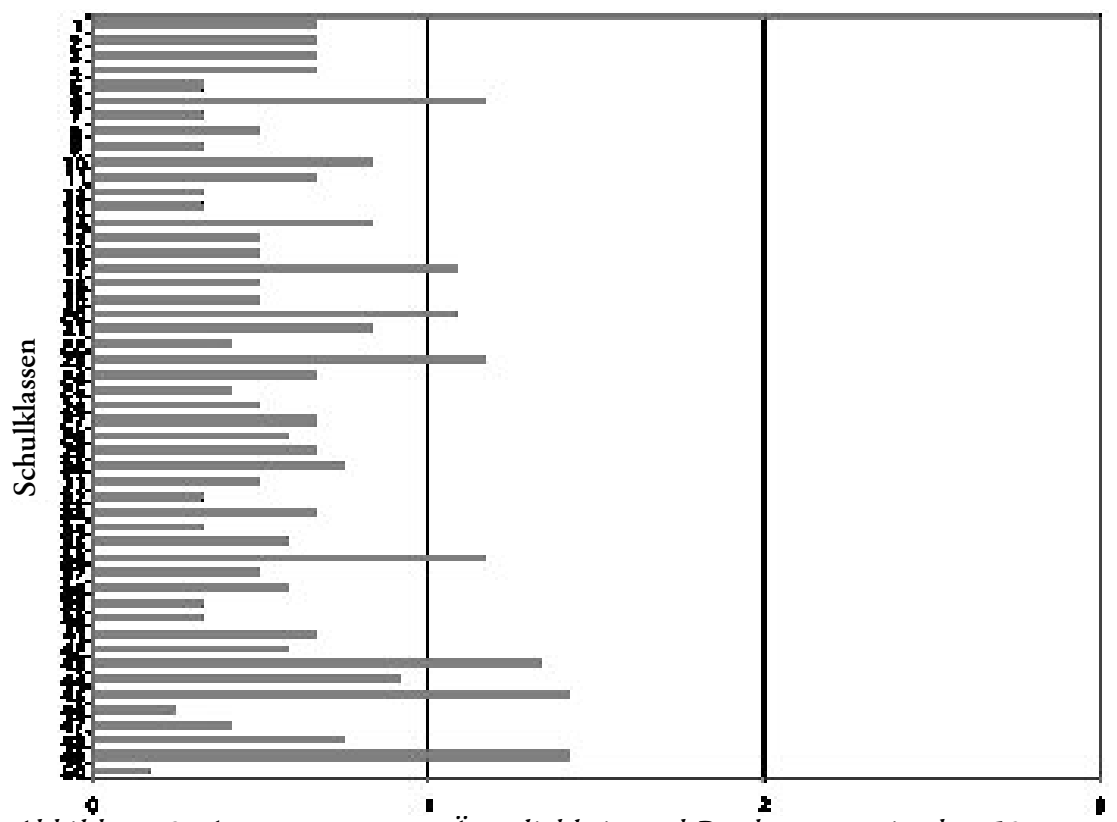

Abbildung 3: Ausprägung von "Ängstlichkeit und Beschämung" in den 50 Schulklassen.

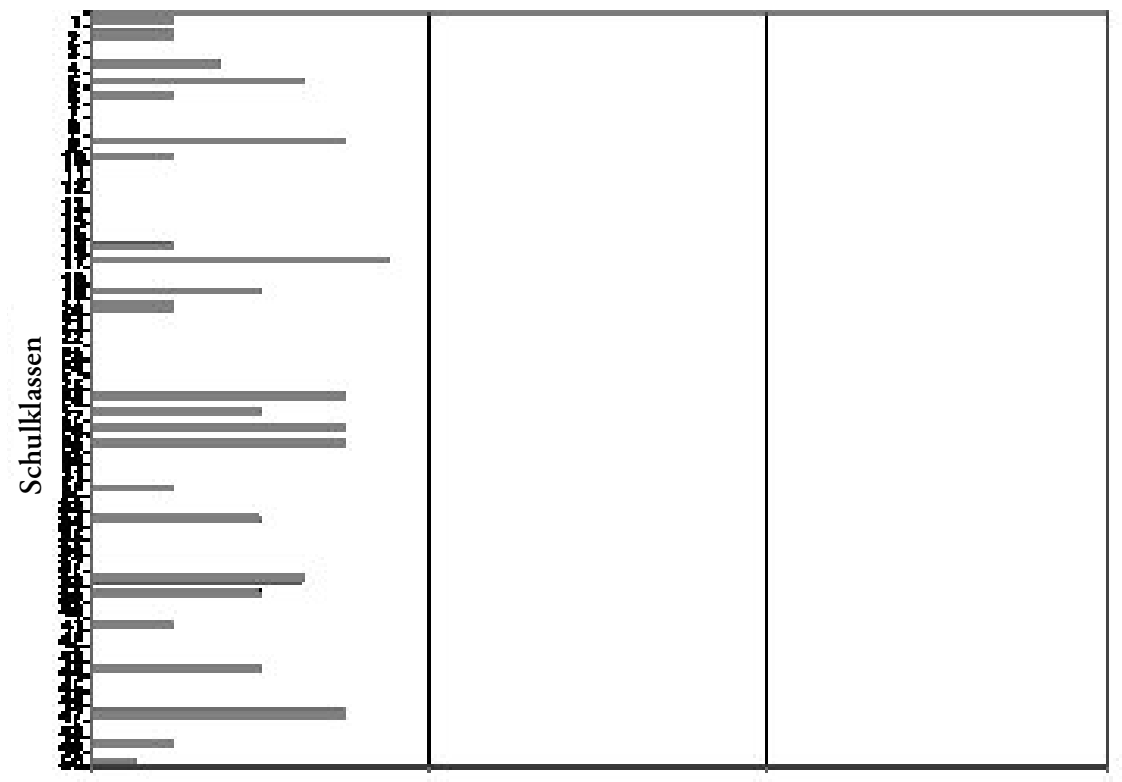

Abbildung 4: Ausprägung von «Fehlervermeidung und negative Fehlerreaktion» in den 50 Schulklassen. 
Besonders niedrig ausgeprägt ist die Ängstlichkeit und Beschämung bei den Klassen 46 und 50. Bei 14 Klassen liegen die Einschätzungen über dem Mittelwert. Besonders hoch ist sie beispielsweise in Klasse 49.

Anders verteilen sich die Einschätzungen der Kodiererinnen für den Aspekt der Fehlervermeidung und der negativen Reaktion auf Fehler. Grundlage für die Kodierungen bildete hier die Bedingung, dass negative Reaktionen auf Fehler bzw. ein auffälliges Vermeiden von Fehlern vorkamen. Figur 4 verdeutlicht, dass in vielen Klassen keine der beiden genannten Aspekte beobachtet und damit die Kodierung $0=$ trifft nicht zu vergeben wurde.

Insgesamt handelt es sich um 24 Klassen, in denen in beiden Stunden keine verächtlichen Reaktionen auf Fehler sowie keine auffällige Fähigkeitsdemonstration auftrat. In den 26 Klassen, in denen eine besondere Fähigkeitsdemonstration bzw. verächtliche Reaktionen auf Fehler in der Klasse vorkamen, zeigt sich darüber hinaus, dass die Ausprägungen insgesamt als niedrig eingestuft wurden (Maximum von 0.88). Damit bestätigen sich bisherige Befunde zum Auftreten von Fehlern im deutschen Unterricht in der Art, dass auffällige Fehlersituationen insgesamt selten bzw. negative Reaktionen darauf in einer geringen Intensität vorkommen.

Zusammenfassend lässt sich festhalten, dass sich das Videoanalyseinstrument aus methodischer Sicht als sehr zuverlässig erwiesen hat und vor allem im Bereich der "Ängstlichkeit und Beschämung» Unterschiede zwischen den Klassen gut abgebildet werden können. Aus diesem Grund werden wir diesen Bereich im Folgenden vertiefen. Es wird dazu nun die Perspektive der Schülerinnen und Schüler in den Mittelpunkt gestellt und der Frage nachgegangen, inwieweit die Ergebnisse der Videoanalysen auch über die Schülereinschätzungen gestützt sind.

\section{Videoanalysen und Schülerselbsteinschätzungen}

Für den Aspekt «Ängstlichkeit und Beschämung» wird das Ergebnis der Videoanalyse mit den Einschätzungen unterstützender Bedingungen durch die Schülerinnen und Schüler kombiniert. Dies hat zum Ziel, mögliche Zusammenhänge zwischen der Ausprägung von «Ängstlichkeit und Beschämung» und den Einschätzungen der unterrichtlichen Lehr-Lernbedingungen zu erkennen und mehr über die Rolle der Vermischung von Lern- und Leistungssituationen für das Lernen im Unterricht zu erfahren.

Die Einschätzungen der Videokodiererinnen und die der Schülerinnen und Schüler korrelieren durchweg positiv (Meyer, in Vorb.). Damit stützen die Schülereinschätzungen die Ergebnisse der Videoanalysen. Schülerinnen und Schüler aus Klassen mit einem ängstlichen Lernklima (ermittelt über Videoanalysen) erleben sich selbst im Unterricht als wenig unterstützt. Dabei unterscheiden sich die Einschätzungen der Schülerinnen und Schüler auch nicht, wenn das Vorwissen der Lernenden hinzugezogen wird. Eine Vermutung könnte darin bestehen, dass nur schwache Schülerinnen und Schüler negativ von schlechten Unter- 
richtsbedingungen beeinflusst werden. Die statistischen Analysen zeigen aber, dass das Vorwissen keineswegs vor der Wahrnehmung von Angst und Beschämung «schützt». Lernende fühlen sich unabhängig vom Ausmass ihres Vorwissens unwohl und wenig unterstützt, wenn sie an einem Unterricht teilnehmen, der durch Angst und Beschämung gekennzeichnet ist. Ebenfalls zeigen sich keine Unterschiede in der Wahrnehmung des Unterrichts zwischen Mädchen und Jungen.

Zusammenfassend lassen sich für den weiteren Einsatz des Schätzverfahrens zwei Ergebnisse festhalten. Anhand der Videokodierungen werden Klassen hinsichtlich des Ausmasses an Ängstlichkeit und Beschämung zuverlässig unterschieden. Die Wahrnehmungen der Schülerinnen und Schüler stützen die Ergebnisse der Videoanalysen.

\section{Vertiefende Analysen an zwei Fallbeispielen}

Ein detaillierter Blick in zwei Unterrichtsklassen der Stichprobe soll exemplarisch zeigen, welche Bedeutung die videogestützte Einschätzung von «Angst und Beschämung» haben kann. Für die vertiefenden Analysen wurden zwei Klassen ausgewählt, die sich im Ausmass von "Angst und Beschämung» deutlich unterscheiden. Gleichzeitig handelt es sich um zwei Schulklassen, die auf der Basis äusserer Rahmendaten gut vergleichbar sind. In beiden Fällen handelt es sich um Gymnasialklassen aus dem gleichen Bundesland. Beide Lehrpersonen sind gleichen Alters und unterrichten seit 4 bzw. 7 Jahren. Für die Videoaufzeichnungen wurde von beiden Lehrpersonen das Thema Optik gewählt und zwei Stunden zur Einführung in die «Linsengesetze» unterrichtet. Von allen Unterrichtsphasen nimmt bei beiden Klassen das «Erarbeiten neuer Inhalte» den grössten Anteil der Unterrichtsstunden in Anspruch.

Die Videokodiererinnen haben die beiden Klassen zusammenfassend folgendermassen beschrieben: Für Schule 49 liegt der Schwerpunkt der unterrichtlichen Aktivitäten auf «Lehrervortrag» und «Klassengespräch» Diese werden durch kurze «Schülerexperimentierphasen» unterbrochen. Die Atmosphäre in der Klasse ist eher angespannt. Die Schülerinnen und Schüler antworten zaghaft und eher fragend-unbestimmt nach Aufforderung. Die Lehrperson reagiert ermahnend auf Fehler und übergeht inhaltlich fehlerhafte Äusserungen.

Im Unterricht der Schule 50 treten neben dem «Erarbeiten neuer Inhalte» wiederkehrend Phasen des Zusammenfassens und Organisierens auf. «Schülerarbeitsphasen» finden nicht statt und die unterrichtlichen Aktivitäten werden zum grössten Teil durch Lehrervorträge bestimmt. Die Atmosphäre in der Klasse ist nicht angespannt. Nach Meldung und nach Aufforderung werden kaum zaghafte Antworten gegeben. Falsche Äusserungen treten auf und werden in den Erarbeitungsprozess integriert. Schülerinnen und Schüler stellen Verständnisfragen an die Lehrperson. Die schriftlichen Zusammenfassungen der Kodiererinnen spiegeln sich im Profil der Kodierungen zu den fünf Bereichen wider (Figur 5). 


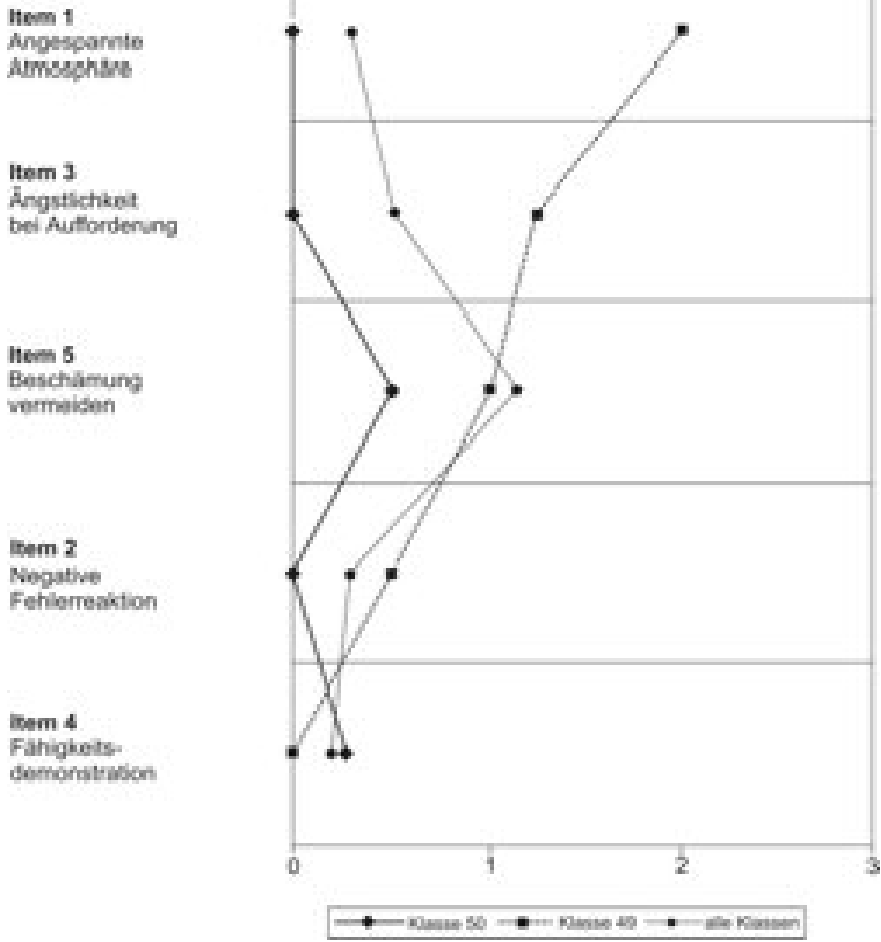

Abbildung 5: Videokodierungen für die Klassen 49 und 50 sowie für die Gesamtsstichprobe (Antwortskala von $0=$ Trifft nicht zu bis 3=Trifft zu).

Für Klasse 49 treten in allen drei Bereichen des Aspektes «Ängstlichkeit und Beschämung» negative Kodierungen auf. Auf Fehler wird in der Klasse negativ reagiert. Eine besondere Demonstration der Fähigkeiten durch die Schülerinnen und Schüler tritt im Unterricht allerdings nicht auf. Die Ergebnisse der Klasse 50 verweisen auf eine nicht angespannte Atmosphäre, in der die Schülerinnen und Schüler insgesamt nur wenig Ängstlichkeit zeigen, vor allem aber dann nicht, wenn sie von der Lehrperson zu einer Äusserung aufgefordert werden. Negative Reaktionen auf Fehler treten nicht auf, vereinzelt stellen Schülerinnen und Schüler ihre Fähigkeiten besonders heraus.

Während sich die groben Muster unterrichtlicher Aktivitäten (lehrer- vs. schülerzentriert) in den beiden Klassen kaum unterscheiden und eher der Klasse 50 günstigere Bedingungen für eine Schülerorientierung zugeschrieben werden könnten, vermag das Videoverfahren zur Vermischung von Lern- und Leistungssituationen Unterschiede zwischen den Klassen abzubilden, die als eine wesent- 
liche Bedingung für die Qualität der Fehlerkultur im Unterricht gelten kann. Welche Rolle die Vermischung von Lern- und Leistungssituationen für das Angebot an Lerngelegenheiten im Unterricht einnehmen könnte, wird im Weiteren exemplarisch durch den Blick auf die von den Schülerinnen und Schülern berichtete Lernmotivation vorgestellt.

Varianten der Lernmotivation in den beiden Schulklassen Zur weiteren Vertiefung der Rolle einer Vermischung von Lern- und Leistungssituationen für das Lernen der Schülerinnen und Schüler stellen wir die in den beiden ausgewählten Klassen berichteten Qualitäten der Lernmotivation vor (Abbildung 6).

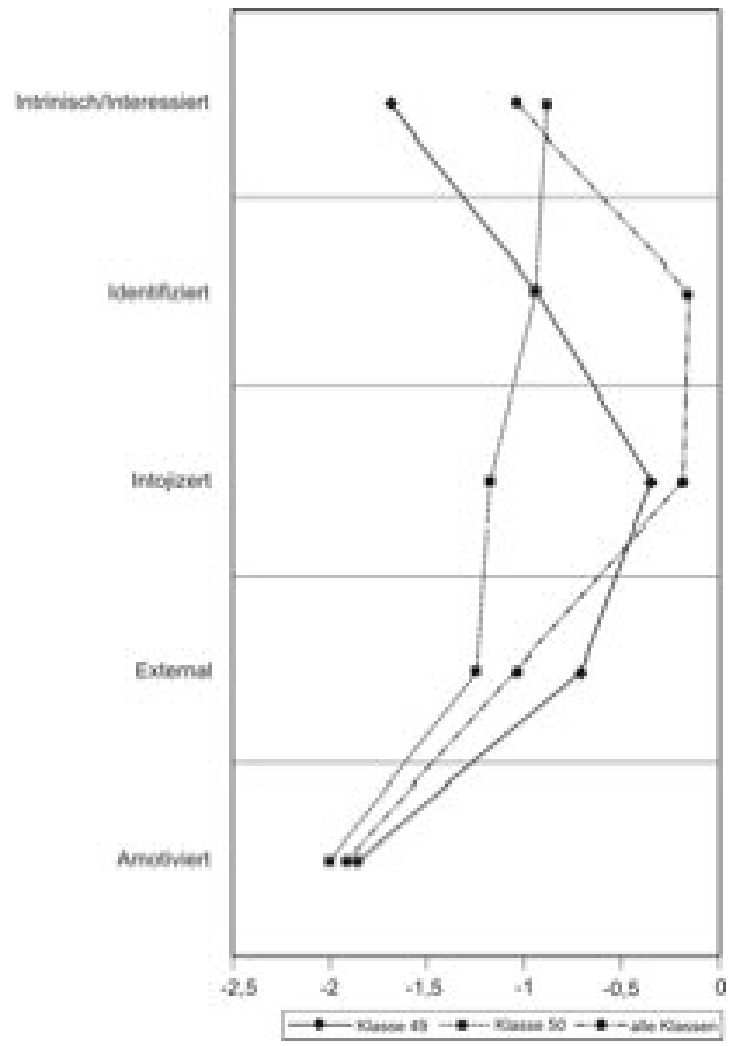

Abbildung 6: Varianten der Lernmotivation in den beiden Schulklassen und im Gesamtmittelwert.

Dazu werden fünf Varianten der Lernmotivation herangezogen, die in der IPN Videostudie über raschanalytische Verfahren überprüft wurden. Die über die Raschanalysen ermittelten Parameter für die einzelnen Schülerinnen und Schüler 
sind hierbei um den Nullpunkt zentriert und variieren bei den Klassen zwischen dem negativen Bereich und dem Nullwert. Während amotiviertes, external motiviertes und introjiziert motiviertes Lernen Formen fremdbestimmten Lernens darstellen, sind die beiden Varianten einer identifizierten und intrinsisch/interessierten Lernmotivation Beispiele für selbstbestimmtes Lernen. Bisherige Forschungsarbeiten zeigen, dass fremdbestimmte Lernmotivation eher zu negativen Lernergebnissen und einer ungünstigen Interessenentwicklung führt. Darüber hinaus zeigen sich für Formen selbstbestimmter Lernmotivation güns-tige Auswirkungen auf kognitive und motivational-affektive Lernergebnisse.

Vor diesem Hintergrund sind die Ergebnisse der beiden Klassen folgendermassen einzuordnen: Klasse 50 weist ein Profil auf, bei dem die Schülerinnen und Schüler bei den selbstbestimmten Varianten der Lernmotivation relativ betrachtet die positivsten Einschätzungen abgeben. Das Ausmass nimmt kontinuierlich in Richtung fremdbestimmter Varianten ab. Damit weist Klasse 50 eine positive Verteilung der Lernmotivationsvarianten auf. Die meisten Schülerinnen und Schüler berichten, in den videographierten Unterrichtsstunden intrinsisch/interessiert und identifiziert motiviert gelernt zu haben. Anders verhält sich das Profil für Klasse 49. Hier zeigen sich besonders niedrige Einschätzungen für intrinsisch/interessierte Lernmotivation und auffällig hohe Werte für ein introjiziertes und external motiviertes Lernen. External motiviert wird ein Lernen dann bezeichnet, wenn es nur aufgrund von äusserem Druck (z.B. von der Lehrperson) erfolgt. Beim introjiziert motivierten Lernen verlagern die Lernenden dieses Drucksystem nach innen und lernen, weil es sich beispielsweise «für eine gute Schülerin oder einen guten Schüler so gehört». Lernen findet aber bei diesen Schülerinnen und Schülern nicht statt, weil kein Anreiz im Lerngegenstand selbst oder in der Lerntätigkeit gesehen wird.

Diese beiden Beispiele verdeutlichen, dass die über Videoanalysen ermittelten Einschätzungen zur Vermischung von Lern- und Leistungssituationen durchaus Relevanz für das Lernen der Schülerinnen und Schüler besitzen. Allerdings stellen sie nur zwei einzelne Beispiele für dieses Zusammenhangsmuster dar. Weitere Analysen werden deshalb derzeit durchgeführt, um das Zusammenspiel zwischen Lehr- und Lern-Prozessen und der Rolle der Fehlerkultur vertiefend zu untersuchen (Meyer, in Vorb.).

\section{Diskussion und Ausblick}

Der vorliegende Beitrag liefert ein Beispiel dafür, wie Aspekte einer Fehlerkultur systematisch mittels Videoanalysen erfasst und Unterschiede zwischen Schulklassen abgebildet werden können. Im Zentrum der theoretischen Überlegungen stand die in Deutschland häufig problematisierte Vermischung von Lernund Leistungssituationen. Um das Ausmass einer Vermischung von Lern- und Leistungssituationen mittels Videoanalysen zu erfassen, wurden solche Aspekte 
im Unterricht in den Blick genommen, die Ausdruck einer gemeinsam geteilten Unterrichtskultur sind. Das Ziel bestand dabei nicht darin, über die Analyse einzelner Unterrichtsaufzeichnungen Ursache-Wirkungs-Zusammenhänge für den Ablauf und das Zustandekommen einer Fehlerkultur zu untersuchen. Im Zentrum der Analysen stand vielmehr die Frage, wie bei einer eingeschränkten Zahl von Unterrichtsaufzeichnungen einer Schulklasse möglichst zuverlässige und aussagefähige Indikatoren für das Vorhandensein einer Vermischung von Lernund Leistungssituationen gewonnen werden können. Wesentliche Grundlage für diese Annahme bilden bisherige Ergebnisse der IPN Videostudie, die zeigen, dass die Analyse einzelner Unterrichtsstunden einen prognostischen Wert für den Ablauf weiterer Unterrichtsstunden hat, insbesondere dann, wenn Interaktionsabläufe zwischen Lehrenden und Lernenden in den Blick genommen werden (Seidel \& Prenzel, 2006). Für eine videogestützte Erfassung der Vermischung von Lern- und Leistungssituationen bedeutete dies, das Bedingungsgeflecht einer Vermischung von Lern- und Leistungssituationen als Ganzes in den Blick zu nehmen und auf die Analyse von Ursache-Wirkungszusammenhängen zu verzichten. Gleichzeitig bestand die Annahme darin, dass dieses Geflecht Ausdruck einer über einen längeren Zeitraum entwickelten Fehlerkultur ist und Relevanz für das weitere Lernen der Schülerinnen und Schüler besitzt.

Die Ergebnisse der Videoanalysen haben gezeigt, dass die Erfassung mittels Beobachtung von videografiertem Unterricht in den beiden ausgewählten Schwerpunkten «Ängstlichkeit und Beschämung» und «Fehlervermeidung und negative Fehlerreaktion» gelungen ist. Internationale Kriterien für die Zuverlässigkeit der Verfahren wurden in allen Bereichen erfüllt. Darüber hinaus haben die statistischen Auswertungen gezeigt, dass die Videoanalysen über die Sicht der Schülerinnen und Schüler gestützt werden. Damit liegt ein für Lehrpersonen durchweg interessantes Instrumentarium bereit. Die Schülereinschätzungen liefern ein differenziertes Meinungsbild der Klasse, die Videoanalysen bereichern diese Einschätzungen über konkrete Unterrichtssituationen, und anhand der konkreten Unterrichtssituationen eröffnen sich wiederum reichhaltige Möglichkeiten für vertiefende Analysen. Die beiden vorgestellten Fallanalysen unterstützen das Potenzial dieser Kombination von Aussen- und Innensicht.

Die deskriptiven Auswertungen verweisen auf weitere Unterschiede zwischen den einbezogenen Schulklassen, allerdings nur für den Bereich der «Ängstlichkeit und Beschämung». Im zweiten Bereich, der «Fehlervermeidung und negativen Fehlerreaktion» werden ähnlich zu früheren Befunden (Heinze, 2004) nur geringe Ausprägungen und Unterschiede zwischen Schulklassen beobachtet. Aus methodischer Sicht bereitet das «Nicht-Auftreten» solcher Situationen erhebliche Schwierigkeiten. Auch wenn diese Aspekte aus theoretischer Sicht von besonderer Relevanz sind, lassen sie sich empirisch in ihrer Bedeutung für das Lernen der Schülerinnen und Schüler nur schwer nachweisen. In dieser Hinsicht erweisen sich Zufallsstichproben bzw. repräsentative Unterrichtsstunden als hin- 
derlich, wenn wie im Bereich der Fehlerkultur Bedingungen herrschen, in denen Fehler systematisch vermieden werden und nicht vorkommen. Forschungsdesigns, in denen systematisch unterschiedliche Fehlerkulturen variiert werden, können hier helfen, theoretische Annahmen über empirische Nachweise zu stützen.

\section{Literatur}

Bortz, J. \& Döring, N. (2002). Forschungsmethoden und Evaluation. Berlin: Springer.

Büeler, U. (2000). In der Klasse stehen. Eine Untersuchung zur nonverbalen Kommunikation der Lehrperson, wenn eine Schülerin oder ein Schüler im schulischen Unterricht einen Fehler macht. Universität Freiburg, CH: Unveröffentlichte Lizentiatsarbeit.

Clausen, M., Reusser, K. \& Klieme, E. (2003). Unterrichtsqualität auf der Basis hoch-inferenter Unterrichtsbeurteilungen: Ein Vergleich zwischen Deutschland und der Schweiz. Unterrichtswissenschaft, 31(2), 122-141.

Götz, T., Zirngibl, A. \& Pekrun, R. (2004). Lern- und Leistungsemotionen von Schülerinnen und Schülern. In T. Hascher (Hrsg.), Schule positiv erleben. Ergebnisse und Erkenntnisse zum Woblbefinden von Schülerinnen und Schülern (S. 49-66). Bern: Haupt.

Hascher, T. \& Oser, F. (1996). Lernen, Motivation und Sozialklima. In C. Spiel (Hrsg.), Motivation und Lernen aus lebenslanger Perspektive. Münster: Waxmann.

Hascher, T. (2004). Wohlbefinden in der Schule. Münster: Waxmann.

Heinze, A. (2004). Zum Umgang mit Fehlern im Unterrichtsgespräch der Sekundarstufe I Theoretische Grundlegung, Methode und Ergebnisse einer Videostudie. Journal für Mathematikdidaktik, 25, 221-244.

Kobarg, M. (2004). Die Bedeutung prozessorientierter Lernbegleitung für kognitive und motivationale Prozesse im Physikunterricht - eine Videostudie. Kiel: IPN, Unveröffentlichte Diplomarbeit.

Kobarg, M. \& Seidel, T. (2005). Coding manual - Process-oriented teaching. In T. Seidel, M. Prenzel \& M. Kobarg (Hrsg.), How to run a video study: Technical report of the IPN Video Study. Münster: Waxmann.

Kobi, E. E. (1994). Fehler. Die neue Schulpraxis, 2, 5-10.

Krapp, A. (2002). An educational-psychological theory of interest and its relation to self-determination theory. In E. L. Deci \& R. M. Ryan (Hrsg.), The handbook of self-determination research (S. 405-427). Rochester: University of Rochester.

Kuntze, S. \& Reiss, K. (2004). Unterschiede zwischen Klassen hinsichtlich inhaltlicher Elemente und Anforderungsniveaus im Unterrichtsgespräch beim Erarbeiten von Beweisen. Unterrichtswissenschaft, 32, 357-379.

Meyer, L. (in Vorb.). Fehlerkultur im Physikunterricht - Anteile und Bedeutungen "negativen» Wissens in der Begriffsbildung.

Oser, F. \& Hascher, T. (1997). Lernen aus Fehlern. Zur Psychologie «negativen» Wissens. Schriftenreihe zum Projekt "Lernen Menschen aus Fehlern? Zur Entwicklung einer Fehlerkultur in der Schule» (Nr. 1). Freiburg: Pädagogisches Institut der Universität Freiburg, CH.

Oser, F. \& Spychiger, M. (2005). Lernen ist schmerzhaft. Zur Theorie des Negativen Wissens und zur Praxis der Fehlerkultur. Weinheim: Beltz.

Pekrun, R. (2000). A Social-Cognitive, Control-Value Theory of Achievement Emotions. In J. Heckhausen (Hrsg.), Motivational Psychology of Human Development. Developing Motivation and Motivating Development (S. 143-163). Amsterdam: Elsevier.

Rimmele, R., Seidel, T., Knierim, B., Kobarg, M., Dalehefte, I. M., Schwindt, K. \& Meyer, L. (2005). Scale documentation - Student questionnaire. In T. Seidel, M. Prenzel \& M. Kobarg (Hrsg.), How to run a video study: Technical report of the IPN Video Study. Münster: Waxmann. 
Schoy-Lutz, M. (2005). Fehlerkultur im Mathematikunterricht. Theoretische Grundlegung und evaluierte unterrichtspraktische Erprobung anhand der Unterrichtseinheit "Einführung in die Satzgruppe des Pythagoras". Hildesheim: Verlag Franzbecker.

Schulmeiss, I. (2004). Vermischung von Lern- und Leistungssituationen und ihre Bedeutung für die Qualität der Lernmotivation. Eine Videostudie im Physikunterricht. Kiel: IPN, Unveröffentlichte Diplomarbeit.

Schulmeiss, I., Seidel, T. \& Meyer, L. (2003). Vermischung von Lern- und Leistungssituationen im Physikunterricht. In T. Seidel, M. Prenzel, R. Duit \& M. Lehrke (Hrsg.), Technischer Bericht zur Videostudie "Lehr-Lern-Prozesse im Physikunterricht» (S. 229-238). Kiel: IPN.

Seidel, T. (2003). Lehr-Lernskripts im Unterricht. Münster: Waxmann.

Seidel, T. \& Prenzel, M. (2003). Mit Fehlern umgehen - Zum Lernen motivieren. Praxis der Naturwissenschaften - Physik in der Schule, 52, 30-34.

Seidel, T., Prenzel, M., Duit, R. \& Lehrke, M. (2003). Technischer Bericht zur Videostudie "Lehr-Lern-Prozesse im Physikunterricht». Kiel: IPN.

Seidel, T. \& Prenzel, M. (2004). Muster unterrichtlicher Aktivitäten im Physikunterricht. In J. Doll \& M. Prenzel (Hrsg.), Bildungsqualität von Schule: Lehrerprofessionalisierung, Unterrichtsentwicklung und Schülerförderung als Strategien der Qualitätsverbesserung (S. 177194). Münster: Waxmann.

Seidel, T. \& Prenzel, M. (2006). Stability of teaching patterns in physics instruction: Findings from a video study. Learning and Instruction, forthcoming. Learning and Instructions, 2 (in press).

Spychiger, M., Oser, F., Hascher, T. \& Mahler, F. (1997). Untersuchung und Veränderung von Fehlerkultur in der Schule: Der Situationsansatz. Schriftenreihe zum Projekt «Lernen Menschen aus Fehlern? Zur Entwicklung einer Fehlerkultur in der Schule» (Nr. 2). Freiburg: Pädagogisches Institut der Universität Freiburg, CH.

Spychiger, M., Oser, F., Hascher, T. \& Mahler, F. (1999). Entwicklung einer Fehlerkultur in der Schule. In W. Althof (Hrsg.), Fehlerwelten. Vom Fehlermachen und Lernen aus Fehlern (S. 43-70). Opladen: Leske + Budrich.

Spychiger, M. (2003). Fehler als Fenster auf den Lernprozess. Zur Entwicklung einer Fehlerkultur in der Praxisausbildung. Journal für Lehrerinnen- und Lehrerbildung, 2, 31-38.

Weinert, F. E. (1999). Aus Fehlern lernen und Fehler vermeiden lernen. In W. Althof (Hrsg.), Fehlerwelten. Vom Fehlermachen und Lernen aus Fehlern (S. 101-109). Opladen: Leske + Budrich.

Zapf, D., Frese, M. \& Brodbeck, F. C. (1999). Fehler und Fehlermanagement. In C. Hoyos \& D. Frey (Hrsg.), Arbeits- und Organisationspsychologie. Ein Lehrbuch. Weinheim: Beltz.

Schlagworte: Videoanalyse, Lernklima, Vermischung von Lern- und Leistungssituationen, Fehlerkultur im Physikunterricht, Motivation, SchülerInnenperspektive 


\section{Quand les situations d'apprentissage deviennent propices aux performances : enquête sur la culture de l'erreur par une étude vidéo.}

\section{Résumé}

Cette contribution montre comment il est possible, par une analyse vidéo, de saisir de manière systématique les différents aspects de la culture de l'erreur et de rendre compte des différences entre classes d'une même école. La confusion souvent discutée en Allemagne entre situation d'apprentissage et situation de restitution des apprentissages est au centre de la recherche. Les résultats de l'analyse vidéo montrent que les critères internationaux de fiabilité sont respectés dans tous les domaines de la procédure et sont en plus étayés par le point de vue des élèves. On présente ainsi un instrumentaire qui identifie un aspect de la complexité des conditions de la culture de l'erreur et des situations concrètes et relevantes d'apprentissage, ouvrant de belles perspectives pour des analyses approfondies. Deux analyses de cas illustrent le potentiel de ce dispositif vidéo.

Mots clés: Analyse vidéo, climat d'apprentissage, confusion des situations d'apprentissage et de restitution des apprentissage, culture de l'erreur dans l'enseignement de la physique, motivation.

\section{Dall'apprendimento alla prestazione: analisi della cultura dell'errore sulla base di un'inchiesta basata su registrazioni video}

\section{Riassunto}

Il contributo mostra come attraverso l'analisi di registrazioni video si possano mostrare in modo sistematico aspetti della cultura dell'errore ed illustrare le diversità tra differenti classi di scuola. Al centro dell'attenzione vi è la sovrapposizione di situazioni di apprendimento e di situazioni in cui è richiesta una prestazione; un problema, questo, assai discusso in Germania. I risultati delle analisi dei video mostrano come siano stati rispettati i criteri internazionali per l'affidabilità delle procedure adottate e come vi sia pure una validazione da parte degli allievi. Disponiamo così di una strumentazione che permette di evidenziare gli aspetti della cultura dell'errore in situazione d'insegnamento significative registrate tramite video. L'illustrazione di due casi mostra il potenziale insito negli strumenti proposti.

Parole chiave: analisi video, clima di classe, sovrapposizione di situazioni di apprendimento e di prestazione, cultura dell'errore nell'insegnamento della fisica, motivazione 


\section{When Learning Situations turn into Achievement Situations: Investigation of Mistake Culture in a Video Study}

\section{Summary}

In this article we investigated how facets of mistake culture can be systematically measured by video analysis and to what extent physics classrooms differed in the extent of turning learning situations into achievement situations. The blending of learning and achievement situations is an intensively discussed problem area in the culture of teaching in Germany. The findings of this article showed that our video rating instrument proved useful in reliably measuring blended learning and achievement situations. In addition, the findings of the video analysis were corroborated by the students' individual perception of learning conditions in the classrooms. Thus, a video analysis instrument is available that can be used to pinpoint the mistake culture in a classroom on the basis of video material. Moreover, the approach offers multiple opportunities for aditional in-depth analyses. In this article, we conclude by illustrating some of these possibilities in two case studies.

Keywords: Video analysis, learning climate, blending of learning and achievement situations, mistake culture in physics instruction, motivation 
$\mathrm{T} \mathrm{h}$ e $\mathrm{m} \mathrm{a}$ 